\title{
EFICACIA DE LA INTERVENCIÓN LINGÜÍSTICA EN ADOLESCENTES CON SÍNDROME DE DOWN
}

\section{Effectiveness of linguistic intervention in adolescents with Down syndrome}

\author{
Lucía Martín-URda Rodríguez \\ Universidad de Castilla La Mancha \\ Teresa Carchenilla Martín \\ Universidad de Castilla La Mancha \\ Esther Moraleda Sepúlveda \\ Universidad de Castilla La Mancha. Av. Real Fábrica de Sedas, s/n. 45600 Talavera de la Reina. \\ Toledo \\ luciamur96@gmail.com
}

Recepción: 15 de noviembre de 2017

Aceptación definitiva: 17 de mayo de 2018

Resumen: El síndrome de Down (SD) es considerado la discapacidad intelectual más conocida y frecuente. El desarrollo cognitivo se produce, normalmente, de manera similar a la secuencia producida en el desarrollo típico; mientras que el desarrollo lingüístico presenta retraso respecto al mismo. Sin embargo, cabe esperar que el desarrollo del lenguaje se beneficie de la intervención logopédica durante la adolescencia y la etapa adulta. El objetivo del presente estudio es conocer si existen mejoras en las áreas del lenguaje oral con una intervención no sistematizada tras el paso del tiempo ( 5 años) en áreas concretas del lenguaje: morfosintaxis, semántica y pragmática. Se ha realizado un estudio longitudinal con dos momentos temporales (2012/2017). La muestra estuvo compuesta por 12 de los sujetos con SD, con edades entre los 13 y los 21 años. Para la evaluación de las áreas del lenguaje se usó la prueba estandarizada Batería del Lenguaje Objetiva y Criterial (BLOC-C), en sesiones individuales. Los resultados obtenidos muestran que no existen diferencias lingüísticas significativas tras cinco años de intervención lingüística realizada por logopedas en ninguna de las áreas evaluadas. Teniendo en cuenta los 
datos obtenidos, es necesario diseñar programas de intervención sistematizados y basados en la evidencia científica que mejoren las características lingüísticas de adolescentes con SD, además de reflexionar sobre las intervenciones que se llevan a cabo actualmente.

Palabras clave: síndrome de Down; lenguaje; intervención logopédica; adolescencia.

Aвstract: Down syndrome (DS) is considered the best known and most frequent intellectual disability. Cognitive development usually occurs in a similar way to the sequence produced in typical development; while the linguistic development is lagging behind it. However, it is expected that language development will benefit from logopedic intervention during adolescence and adulthood. The objective of the present study is to know if there are improvements in the areas of oral language with a non-systematized intervention after the passage of time (5 years) in specific areas of language: morphosyntax, semantics and pragmatics. A longitudinal study was carried out with two temporal moments (2012/2017). The sample consisted of 12 of the subjects with DS, aged between 13 and 21 years. For the evaluation of language areas, the standardized Objective and Criteria Language Battery test (BLOC-C) was used in individual sessions. The results obtained show that there are no significant linguistic differences after five years of linguistic intervention performed by speech therapists in any of the areas evaluated. Taking into account the data obtained, it is necessary to design intervention programs systematized and based on scientific evidence that improve the linguistic characteristics of adolescents with DS, in addition to reflecting on the interventions that are currently carried out.

KEY WORDS: Down syndrome; language; speech therapy intervention; adolescence.

\section{Introducción}

$\mathrm{E}$

L SíndRome De Down (en adelante SD) es la discapacidad intelectual de origen genético más conocida y frecuente, representando aproximadamente al 25-30\% de las personas con discapacidad intelectual (Galeote, Soto, Sebastián, Rey y Checa, 2014; Moreno y Díaz, 2014; Santos y Bajo, 2011), con una prevalencia entre el 6,1 y el 13,1 por cada 10.000 personas (Bertapelli, Pitetty, Agiovlasitis y Guerra, 2016).

El desarrollo cognitivo en el SD sigue un curso que generalmente es el típico de la secuencia del desarrollo normal. No obstante, existen amplias diferencias individuales en el ritmo de desarrollo. Por ejemplo, el Cociente Intelectual (CI) tiene un promedio de 50, pero con variaciones entre 30 y 70 (Chapman y Hesketh, 2000). Estas diferencias se producen con unas áreas específicas de retraso importante, como en el caso del lenguaje expresivo y la memoria verbal a corto plazo (Chapman, 1997).

En relación a las características lingüísticas, las personas con SD también presentan un claro retraso con respecto a las personas con desarrollo típico (Beeghly y Cicchetti, 1986, 1990; Oliver y Buckley, 1994), observándose en las diferentes áreas del lenguaje. En el área de semántica, la adquisición de las primeras palabras se encuentra 
retrasada (Abbeduto, Warren y Conners, 2007) y el crecimiento del vocabulario es más lento. Esta adquisición y producción más lenta es observada en niños y adolescentes. Especialmente el vocabulario expresivo se encuentra más retrasado de lo que debería encontrarse en función de su edad mental (Martín, Klusek, Estigarribia y Roberts, 2009). Sin embargo, el léxico es considerado un punto fuerte en las personas con SD (Galeote, Soto, Checa, Gómez y Lamela, 2008; Kay-Raining Bird, Gaskell, Dallaire y Macdonald, 2000; Martin et al., 2009; Miller, 1992; Miller, Leddy y Leavitt, 2001; Singer-Harris, Bellugi, Bates, Jones y Rosen, 1997). En la infancia y la adolescencia, la comprensión del vocabulario es un área de relativa fortaleza de las personas con SD. En contraste con otros dominios de la lengua, la comprensión del vocabulario mantiene el ritmo o incluso supera a los componentes no verbales en desarrollo cognitivo en adolescentes y jóvenes con SD (Abbeduto et al., 2003; Chapman, Schwartz y Kay-Raining Bird, 1991; Rosin Swift, Bless y Vetter, 1988). Algunos resultados con el Test de Vocabulario en Imágenes Peabody (TVIP), comparando el conocimiento de vocabulario pasivo de un grupo de personas con SD de entre 12 y 21 años con el de un grupo de niños con desarrollo típico de la misma edad mental, encuentran que ambos grupos muestran un buen rendimiento, que mejora con la edad (Chapman, 2006). De este modo, podría sostenerse que la relativa preservación del repertorio léxico queda dentro del perfil específico del SD.

En cuanto a las características morfosintácticas, parecen presentar una gran dificultad en las personas con SD (Abbeduto, Warren y Conners, 2007; Moraleda, 2011), ya que existen estudios que muestran que esta área se encuentra más deteriorada que el vocabulario (Abbeduto et al., 2003; Berglund y Eriksson, 2000; Chapman et al., 1991; Laws y Bishop, 2003; Martín, Klusek, Estigarribia y Roberts, 2009). La comprensión sintáctica en todas las edades de las personas con SD (niños, adolescentes y adultos) es menor de lo que se espera conforme a su capacidad cognitiva no verbal, disminuyendo la capacidad comprensiva de esta área a medida que aumenta la edad (Vicari, Caselli y Tonucci, 2000). Las combinaciones de dos palabras se producen de forma más lenta y retrasada. Este retraso hace que los niños y adolescentes produzcan enunciados cortos y de menor complejidad (Chapman, Hesketh y Kistler, 2002; Thordardottir, Chapman y Wagner, 2002). De esta manera, suelen usar frases telegráficas más simples y suelen tener dificultades con la morfología (Vicari, Caselli y Tonucci, 2000). Parece, sin embargo, que las capacidades morfosintácticas pueden desarrollarse en la adolescencia y la edad adulta, con una correcta intervención (Chapman, Hesketh y Kistler, 2002; Thordardottir, Chapman y Wagner, 2002).

Por último, en relación al área pragmática, se observan un retraso en la aparición de la comunicación intencional, así como retraso en la madurez de sus enunciados. Las personas con SD usan las funciones pragmáticas de la misma manera que los niños de su edad, aunque aparecen de forma retrasada (Channell, McDuffie, Bullard y Abbeduto, 2015). Sin embargo, una vez que utilizan el habla como medio de comunicación usan el lenguaje de manera social como los niños con desarrollo típico (Abbeduto et al., 2008). Los niños con SD suelen ser persistentes en los temas de conversación y pueden tener dificultades en iniciar las conversaciones con temas menos frecuentes, expresándose de forma menos clara (Abbeduto, Warren y Conners, 2007; Martín, Klusek, 
Estigarribia y Roberts, 2009). No obstante, el uso de gestos para su comunicación parece ser un punto fuerte en estos niños (Abbeduto, Warren y Conners, 2007). En las narraciones, son capaces de reconocer y expresar los elementos de estas tareas a pesar de sus limitaciones léxicas y sintácticas, de forma más simple (Abbeduto et al., 2008). Las personas con SD son más eficaces si poseen ayudas visuales, recuerdan más datos sobre la narración y el tema, aunque esta capacidad disminuye con la edad (Abbeduto, Warren y Conners, 2007; Martín, Klusek, Estigarribia y Roberts, 2009). La pragmática es considerada como un punto fuerte en las características de los niños con SD, ya que no suelen tener deterioros pragmáticos graves (Laws y Bishop, 2004).

En cuanto al desarrollo evolutivo del lenguaje, la investigación ha constatado que el aprendizaje del lenguaje en personas con SD ocurre en la niñez y continúa durante la adolescencia (Buckley, 1993; Chapman, 1997; Cossu, Rossini y Marshall, 1993), en contra de lo que formulaban investigaciones anteriores (Lennenberg, 1967; Fowler, 1990). Lejos de las creencias comúnmente aceptadas relacionadas con las edades de aprendizaje, el área morfosintáctica continúa su desarrollo más allá de la infancia y sigue consolidándose en la adolescencia, si se sigue trabajando con un programa de intervención adecuado (Moraleda, Lázaro y Garayzábal, 2013). Parece, pues, que la intervención logopédica sigue siendo efectiva a lo largo de todas las etapas evolutivas (Pelatti, 2015). Sin embargo, aunque muy pocos adultos reciben intervención logopédica, estudios realizados señalan que los adultos pueden mejorar sus habilidades del habla y del lenguaje (Kumin, 2002). Por tanto, dada la escasez de estudios longitudinales con esta población sobre intervención lingüística, el objetivo de este estudio es conocer si existen mejoras en las distintas áreas del lenguaje oral en un grupo de adolescentes con SD a través del paso del tiempo tras recibir una intervención lingüística no sistematizada.

\section{Método}

Se diseñó un estudio longitudinal con una evaluación lingüística en dos momentos temporales (enero de 2012/mayo de 2017) de un grupo de personas con SD: primero, una evaluación inicial; y segundo, una reevaluación tras un periodo de 5 años sin someterse a una intervención lingüística sistematizada, pero sí recibiendo terapia logopédica no sistematizada, acudiendo dos veces por semana en sesiones de 40 minutos. Se entiende por intervención logopédica sistematizada aquella que está regulada a través de un programa de intervención estandarizado como tal. En el caso de este estudio, se considera que la intervención recibida a lo largo de este tiempo ha sido no sistematizada puesto que los participantes no han seguido un protocolo de intervención preestablecido, sino que han recibido intervención del lenguaje en base a los criterios profesionales de los distintos logopedas que trabajaban con ellos.

\subsection{Participantes}

Inicialmente fueron 20 los participantes que accedieron a participar en este estudio, pero finalmente, tras cinco años, se les pudo hacer un seguimiento a un total 
de 12 sujetos con SD. En el primer momento temporal tenían edades comprendidas entre los 7 y 13 años $(X=10,86$; DT $=2,4)$ y en el segundo momento temporal, entre 13 y 19 años $(\mathrm{X}=16,78$; DT $=2,16)$. De esta muestra, 7 participantes son chicos y 5 chicas. También se controló el CI para que no pudieran existir diferencias según el factor inteligencia. En enero de 2012, la media era de $\mathrm{X}=44,58$ (DT $=3,82$ ) y en mayo de 2017, $\mathrm{X}=44,75$ (DT $=3,41)$, no existiendo diferencias significativas entre ambas fechas.

\subsection{Instrumentos}

Dado que la capacidad intelectual en los niños con SD es muy variable, y esta capacidad podría explicar el distinto rendimiento antes y después de la aplicación del protocolo, se evaluó la misma a través de la escala de inteligencia WISC-IV (Weschler, 2003). Existen estudios que demuestran la fiabilidad y validez de esta escala en personas con SD (Braden y Hanna, 1998; Canivez y Walkins, 1998; Hishinuma, 1998). Es una de las pruebas utilizadas con más frecuencia para evaluar aspectos cognitivos de las personas con discapacidad intelectual (AAMR, 2002).

Para llevar a cabo la evaluación lingüística se utilizó la versión completa de la prueba estandarizada Batería del Lenguaje Objetiva y Criterial, en su versión informatizada (BLOC-INFO) (Puyuelo, Renom, Solanas y Wiig, 2007). Esta prueba se administra a poblaciones comprendidas entre 5 y 14 años de edad, aunque está adaptada para poblaciones con necesidades especiales. Evalúa 4 aspectos del lenguaje: morfología, sintaxis, semántica y pragmática. Compuesta por un total de 580 ítems divididos de la siguiente forma: 190 ítems de morfología, 180 ítems que evalúan sintaxis, 80 ítems relacionados con la semántica y un total de 130 ítems para evaluar pragmática. La versión informatizada proporciona la corrección al instante y elabora gráficas del perfil obtenido en las diferentes áreas, permitiendo guardar los resultados obtenidos, así como las respuestas. Además, los resultados de esta prueba pueden ser comparados con los resultados de otros sujetos con la misma edad cronológica o mental.

\subsection{Procedimiento}

Primero, se contactó con una asociación de SD con el fin de conocer si estarían interesados en que se llevara a cabo este estudio durante estos cinco años. Una vez que la asociación accedió y se confirmaron los sujetos de la muestra, se les envió el documento de consentimiento informado. Tras la firma de dicho documento, se llevó a cabo la evaluación del lenguaje individualizada mediante WISC-IV y el BLOC.

La evaluación del lenguaje en los dos momentos temporales fue realizada por un logopeda especializado en SD. Fueron necesarias dos sesiones en cada momento para llevar a cabo la evaluación del lenguaje. Es importante mencionar que todos los ítems fueron administrados en el orden establecido por el test y que a todos los sujetos se les dieron las mismas instrucciones indicadas en dicho instrumento. 


\section{Resultados}

En la Tabla 1, en la que se muestran las puntuaciones obtenidas en relación a las dos tomas de datos realizadas, se puede observar cómo las puntuaciones son más bajas en el segundo momento temporal (mayo de 2017) en las áreas de sintaxis, semántica y pragmática, así como que el área de morfología obtiene una puntuación ligeramente superior. No obstante, las diferencias no son significativas en ninguna de las áreas: morfología $(\mathrm{t}(11)=-, 369, \mathrm{p}>, 05)$, sintaxis $(\mathrm{t}(11)=1,32, \mathrm{p}>, 05)$, semántica $(\mathrm{t}(11)=$ $2,19, \mathrm{p}>, 05)$ y pragmática $(\mathrm{t}(11)=1,02, \mathrm{p}>, 05)$.

\begin{tabular}{|l|c|c|}
\hline \multicolumn{3}{|c|}{ Tabla 1. Puntuaciones en las áreas lingüísticas } \\
\hline & Evaluación inicial enero 2012 & Reevaluación mayo 2017 \\
\hline Morfología & $24,91(13,0)$ & $25,67(10,9)$ \\
\hline Sintaxis & $16,16(12,0)$ & $12,16(6,8)$ \\
\hline Semántica & $41,08(19,9)$ & $32,33(9,6)$ \\
\hline Pragmática & $4,41(4,9)$ & $3,25(5,3)$ \\
\hline
\end{tabular}

Desviaciones típicas entre paréntesis.

De igual modo, si analizamos específicamente por áreas, se puede comprobar cómo sí existen diferencias significativas en algunas de las subáreas. Por ejemplo, en morfología se observan mejoras en la formación de plurales acabados en consonante $(\mathrm{t}(11)=-2,96, \mathrm{p}<, 05)$, formas verbales regulares de presente $(\mathrm{t}(11)=-3,02, \mathrm{p}<, 05)$, formas verbales regulares de pasado $(\mathrm{t}(11)=-3,22, \mathrm{p}<, 01)$, formas verbales regulares de futuro $(\mathrm{t}(11)=-2,44, \mathrm{p}<, 05)$ y formas verbales regulares de imperfecto $(\mathrm{t}(11)=$ $-2,34, p<, 05)$. En sintaxis, solo existe mejora en el subárea de oraciones negativas (t $(11)=-2,33, p<, 05)$. Sin embargo, no existen diferencias significativas en ninguna de las subáreas de semántica y pragmática.

\section{Discusión y conclusiones}

Los resultados obtenidos en este estudio contradicen los encontrados por otros estudios realizados previamente que confirmaban la existencia de mejoras en las áreas de morfosintaxis, semántica y pragmática en el periodo adolescente (Chapman, 1997; Kumin, 2002). Por ejemplo, Leddy y Gill (1999) describen avances en el vocabulario, la sintaxis y las habilidades de conversación en los adultos con SD que participaron en las terapias semanales individuales y de grupo. Parece que la intervención en el lenguaje en adolescentes tardíos puede ser beneficiosa, ya que existe una adquisición continua tanto expresiva como comprensiva (Buckley, 1993; Cossu, Rossini y Marshall, 1993). De igual modo, los estudios revisados por Rosenberg y Abbeduto (1993) también afirman la existencia de un desarrollo continuo hasta la edad adulta, aunque sea necesario realizar comparaciones más detalladas. Estos datos, hasta ahora, constituían un aliciente para continuar aplicando terapias de intervención lingüística durante 
las distintas etapas del desarrollo. Sin embargo, es inquietante que, tras cinco años de intervención logopédica realizada por profesionales, no se hayan producido mejoras significativas en ninguna de las áreas lingüísticas y solo se produzcan mejorías en cuanto a formas verbales regulares, morfología de número y el empleo de oraciones negativas. Esto nos lleva a pensar en varias posibilidades. Por un lado, tal y como señala Lennenberg (1967) en un estudio longitudinal con 62 niños con SD de 3 años que compara posteriormente en el periodo adolescente, podría confirmarse la hipótesis del periodo crítico para explicar el hecho de que los que llegan a la adolescencia hubieran fracasado a la hora de lograr cualquier progreso perceptible en la adquisición de estructuras lingüísticas, en contraste con niños más pequeños. Los participantes en el estudio no mostraron mejoras sintácticas significativas más allá de la adolescencia, al igual que ha ocurrido en este estudio. Siguiendo la misma línea, Fowler (1990) no delimita la edad de aprendizaje en la adolescencia, sino que sugiere la existencia de un límite madurativo en el aprendizaje lingüístico hacia los 7 años. Ambos estudios concluyen, por tanto, que no cabe esperar progreso alguno en el desarrollo sintáctico durante la adolescencia de las personas con SD.

No obstante, otra hipótesis que puede explicar los resultados obtenidos es que la intervención que se ha llevado a cabo durante todo este tiempo no ha sido efectiva porque no estaba correctamente diseñada y/o realizada, al tratarse de una intervención no sistematizada. Además, en este punto, sería conveniente referirse al efecto “persona” de cada uno de los terapeutas del lenguaje implicados en este proceso. No se han podido controlar las características personales y profesionales de cada uno de ellos, teniendo en cuenta que quizás las actividades planteadas durante estos cinco años no han sido correctamente elegidas para trabajar sobre aquellas dificultades lingüísticas específicas que presenta este colectivo. El objetivo de los programas de intervención debe ser que estos sean capaces de optimizar la adquisición de habilidades lingüísticas durante la etapa de la adolescencia y quizás sea necesaria una reflexión profunda acerca de la formación específica que se necesitaría para realizarse adecuadamente en el caso del síndrome de Down.

De hecho, de la revisión acerca de los programas de intervención logopédica llevados a cabo previamente con personas con SD se pueden extraer ciertas conclusiones relevantes. La primera conclusión es que aún existe poca investigación basada en la eficacia de los programas y estrategias de intervención que permiten mejorar las características lingüísticas en las personas con SD (Pelatti, 2015; Roberts, Price y Malkin, 2007), lo que nos impide comprobar la eficacia de las mismas. La segunda conclusión es que, tras la revisión efectuada, se observa que la mayoría de los trabajos han sido realizados con niños de habla inglesa y, por ello, los resultados podrían no concordar con los obtenidos en este trabajo. De acuerdo con Vicari et al. (2000), sería necesario investigar con otras lenguas con mayor complejidad, como es el caso del español. En este sentido, creemos que nuestro estudio aporta un valor relevante, pues existían escasas investigaciones hasta el momento en español acerca de la eficacia de la intervención lingüística en personas con SD, donde sí se ha comprobado que un programa de intervención morfosintáctica sistematizado no solo mejora las habilidades morfosintácticas en niños y adolescentes con SD, sino que también lo hace a nivel 
de semántica (Moraleda, Lázaro y Garayzábal, 2013). Por tanto, es necesario seguir insistiendo no solo en la implantación de intervención lingüística con este colectivo, sino en la necesidad de que existan programas de intervención del lenguaje controlados y sistematizados a lo largo de las distintas etapas evolutivas dado que parece que aún en la vida adulta se pueden seguir mejorando ciertos aspectos del lenguaje (Rondal y Buckley, 2003).

\section{Referencias bibliográficas}

Abbeduto, L., Murphy, M. M., Cawthon, S. W., Richmond, E. K., Weissman, M. D., KaRAdotTIR, S. y O’Brien, A. (2003). Receptive language skills of adolescents and young adults with Down or fragile X syndrome. American Journal on Mental Retardation, 108 (3), 149-160.

Abbeduto, L., Murphy, M. M., Kover, S. T., Giles, N. D., Karadottir, S., Amman, A. ... y Nollin, K. A. (2008). Signaling noncomprehension of language: A comparison of fragile X syndrome and Down syndrome. American Journal on Mental Retardation, 113 (3), 214-230.

Abbeduto, L., Warren, S. F. y Conners, F. A. (2007). Language development in Down syndrome: From the prelinguistic period to the acquisition of literacy. Developmental Disabilities Research Reviews, 13 (3), 247-261.

American Association on Mental Retardation (2002). Mental retardation. Definition, classification and systems of supports (10th ed.). Washington: AAMR.

Beeghly, M. y Cicchetti, D. (1986). Early language development in children with Down Syndrome: A longitudinal study. Comunicación presentada en XI Anual Boston University Conference on Language Development, Boston.

Beeghly, M., Weiss-Perry, B. y Cicchetti, D. (1990). Beyond sensoriomotor functioning: early communicative and play development of children with Down syndrome. En D. CicChetTi y M. Beeghly (Eds.), Children with Down Syndrome: A Developmental Perspective (pp. 329-368). Cambridge: Cambridge University Press.

Berglund, E. y Eriksson, M. (2000). Communicative Development in Swedish Children 1628 months old: The Swedish Early Communicative Development Inventory-Words and Sentences. Scandinavian Journal of Psychology, 41, 133-144.

Bertapelli, F., Pitetti, K., Agiovlasitis, S. y Guerra-Junior, G. (2016). Overweight and obesity in children and adolescents with Down syndrome-prevalence, determinants, consequences, and interventions: A literature review. Research in Developmental Disabilities, 57, 181-192.

Braden, J. P. y Hanna, J. M. (1998). Assessment of hearing-impaired and deal children, with the WISC-III. En A. Prifitera y D. H. Saklofske (Eds.), WISC-III clinical use and interpretation: Scientist-practitioner perspectives (pp. 175-201). San Diego: Academic Press.

Buckley, S. (1993). Language development in children with Down syndrome-Reasons for optimism. Down Syndrome Research and Practice, 1 (1), 3-9.

Canivez, G. L. y Walkins, M. W. (1998). Long term stability of the Weschler Intelligence for children, among students with disabilities. Third edition. School Psychology Review, 30 (2), 438-453.

Channell, M. M., McDuffie, A. S., Bullard, L. M. y Abbeduto, L. (2015). Narrative language competence in children and adolescents with Down syndrome. Frontiers in Behavioral Neuroscience, 9, 283. 
Chapman, R. (2006). Language learning in Down syndrome: the speech and language profile compared to adolescents with cognitive impairment of unknown origin. Down Syndrome Research and Practice, 10 (2), 61-66.

Chapman, R. S. (1997). Language development in children and adolescents with Down syndrome. Developmental Disabilities Research Reviews, 3 (4), 307-312.

Chapman, R. S. y Hesketh, L. J. (2000). Behavioral phenotype of individuals with Down syndrome. Developmental Disabilities Research Reviews, 6 (2), 84-95.

Chapman, R. S., Hesketh, L. J. y Kistler, D. J. (2002). Predicting longitudinal change in language production and comprehension in individuals with Down syndrome: Hierarchical linear modeling. Journal of Speech Language and Hearing Research, 45 (5), 902-915.

Chapman, R. S., Schwartz, S. E. y Bird, E. K. R. (1991). Language Skills of Children and Adolescents With Down Syndrome I. Comprehension. Journal of Speech, language and bearing research, 34 (5), 1106-1120.

Cossu, G., Rossini, F. y Marshall, J. C. (1993). When reading is acquired but phonemic awareness is not: A study of literacy in Down's syndrome. Cognition, 46 (2), 129-138.

Fowler, A. (1990). Language abilities in children with Down syndrome: Evidence for a specific syntactic delay. En D. Cicchetti y M. Beeghly (Eds.), Children with Down syndrome: A Developmental Perspective (pp. 302-328). Cambridge: Cambridge University Press.

Galeote, M., Soto, P., Checa, E., Gómez, A. y Lamela, E. (2008). The acquisition of productive vocabulary in Spanish children with Down syndrome. Journal of Intellectual and Developmental Disability, 33 (4), 292-302.

Galeote, M., Soto, P., Sebastián, E., Rey, R. y Checa, E. (2014). La adquisición del vocabulario en niños con síndrome de Down: datos normativos y tendencias de desarrollo. Infancia y Aprendizaje, 35 (1), 111-122.

Hishinuma, E. S. (1998). Issues Related to WAIS-R Testing Modifications for Individuals with Learning Disabilities or Attention-Deficit/Hyperactivity Disorder. Learning Disability Quarterly, 21 (3), 228-240.

Kay-Raining Bird, E., Gaskell, A., Dallaire Babineau, M. y MacDonald, S. (2000). Novel word acquisition in children with Down Syndrome: Does modality make a difference? Journal of Communication Disorders, 33, 241-266.

Kumin, L. (2002). Inteligibilidad del habla en las personas con síndrome de Down: Un marco para señalar factores específicos útiles en la evaluación y tratamiento. Revista Síndrome de Down, 19 (1), 14-23.

LAws, G. y Bishop, D. V. (2003). A comparison of language abilities in adolescents with Down syndrome and children with specific language impairment. Journal of Speech Language and Hearing Research, 46 (6), 1324-1339.

Laws, G. y Bishop, D. V. (2004). Verbal deficits in Down's syndrome and specific language impairment: a comparison. International Journal of Language and Communication Disorders, 39 (4), 423-451.

Lázaro, M., Garayzábal, E. y Moraleda, E. (2013). Differences on morphological and phonological processing between typically developing children and children with Down syndrome. Research in Developmental Disabilities, 34 (7), 2065-2074.

LedDy, M. y Gill, G. (1999). Enhancing the speech and language skills of adults with Down Syndrome. En J. Miller, M. Leddy y L. LeavitT (Eds.), Improving communication of people with Down syndrome (pp. 205-213). Baltimore: Brookes.

Lenneberg, E. (1967). Biological foundation of language. New York: Wiley. 
Martin, G. E., Klusek, J., Estigarribia, B. y Roberts, J. E. (2009). Language characteristics of individuals with Down syndrome. Topics in Language Disorders, 29 (2), 112.

Miller, J. (1992). Development of speech and language in children with Down syndrome. En I. T. LotT y E. E. McCoy (Eds.), Down Syndrome: Advances in Medical Care (pp. 39-50). Chichester: Wiley.

Miller, J., Leddy, M. y LeavitT, L. (2001). Síndrome de Down: comunicación, lenguaje, habla. Barcelona: Masson y Fundación Síndrome de Down de Cantabria.

Moraleda, E. (2011). Análisis del desarrollo morfosintáctico en personas con Síndrome de Down en el periodo infantil y adolescente. Revista de Investigación en Logopedia, 1 (2), 121-129.

Moraleda, E., Lázaro, M. y Garayzábal Heinze, E. (2013). Can individuals with Down syndrome improve their grammar? International Journal of Language E Communication Disorders, 48 (3), 343-349.

Moreno, E. y Díaz, F. (2014). Evaluación del componente pragmático en Síndrome de Down a través del Protocolo Rápido de Evaluación Pragmática. Revista de Investigación en Logopedia, 4 (1), 1-27.

Oliver, B. y Buckley, S. (1994). The language development of children with Down syndrome: First words to two-word phrases. Down Syndrome Research and Practice, 2 (2), 71-75.

Pelatti, C. (2015). Enhancing oral and written language for adolescents and young adults with Down syndrome. Seminars in Speech and Language, 36 (1), 50-59.

Puyuelo, M., Renom, J., Solanas, A. y Wiig, E. (2007). Batería del lenguaje objetiva y criterial. BLOC. Barcelona: Masson.

Roberts, J., Price, J. y Malkin, C. (2007). Language and Communication Development in Down Syndrome. Mental Retardation and Developmental Disabilities Research Reviews, $13,26-35$.

Rondal, J. y Buckley, F. (2003). Speech and language intervention in Down syndrome. London: Whurr.

Rosenberg, S. y Abbeduto, L. (1993). Language and Communication in Mental Retardation: Development, process, and intervention. Hillsdale: Lawrence Erlbaum Associates.

Santos, E. y Bajo, C. (2011). Alteraciones del lenguaje en pacientes afectos de síndrome de Down. Revista ORL, (2), 9.

Singer-Harris, N. G., Bellugi, U., Bates, E., Jones, W. y Rossen, M. L. (1997). Contrasting profiles of language development in children with Williams and Down syndromes. Developmental Neuropsychology, 13 (3), 345-370.

Thordardottir, E. N., Chapman, R. S. y Wagner, L. (2002). Complex sentence production by adolescents with Down syndrome. Applied Psycholinguistics, 23 (2), 163.

Vicari, S., Caselli, M. C. y Tonucci, F. (2000). Asynchrony of lexical and morphosyntactic development in children with Down syndrome. Neuropsychologia, 38 (5), 634-644.

Wechsler, D. (2003). WISC-IV: Technical and interpretive manual. San Antonio: The Psychological Corporation. 